\title{
Optimal location of fast charging stations in Barcelona: A Flow-Capturing approach
}

\author{
M. Cruz-Zambrano, C. Corchero, L. Igualada-Gonzalez \\ Catalonia Institute for Energy Research \\ Jardins de les Dones de Negre, 1 \\ Sant Adrià de Besòs \\ 08930 Barcelona, Spain \\ Email:\{mcruz, ccorchero, ligualada\}@irec.cat
}

\author{
Valeria Bernardo \\ Department of Economic Policy \\ Universitat de Barcelona \\ Avinguda Diagonal 690 \\ 08034 Barcelona, Spain \\ Email: valeriabernardo@ub.edu
}

\begin{abstract}
The aim of this paper is to find the optimal location of electric vehicle (EV) fast charging stations by means of two methodologies: a classical flow-capturing optimization model involving only mobility needs, and an advanced flow-capturing optimization model including distribution network and location costs. While the first model aims to maximize the public service provided by the fast charging stations, the second also considers the incurred cost for providing it. Results from both models are compared in order to analyse the effect of both planning approaches in total cost of installation. As a case study it has been chosen the city of Barcelona.
\end{abstract}

Index Terms-Fast charging stations, distribution network planning, optimal location.

\section{INTRODUCTION}

Electric vehicles have become a strategic technology investment for the automotive sector over the past few years. Currently a significant share of automobile manufacturers produce plug-in electric vehicles (PEV), both battery electric vehicles (BEV) or plug-in hybrid electric vehicles (PHEV). Furthermore, during the next few years almost the major of manufacturers are planning to introduce PEV models. This maybe attributed to: first, the increase of cost-effectiveness of the technology, mainly driven by the reduction of the battery cost and rising fossil fuel prices; second, the environmental and energy policies set up at international level, supported both by geo-strategic reasons as well by public awareness and desire for sustainability.

Recent market penetration scenarios regarding PEV are reducing expectations of previous market analyses because of the global economic situation during of the last years. However electric vehicles are still considered as one of the main technological alternatives for reducing European energy external dependence, as well as urban pollution. Proof of that is the recently published MEMO/13/24, were the European Commission highlights that the availability of recharging/refuelling stations is not only a technical prerequisite for the functioning of electric vehicles, but also one of the most critical components of consumer acceptance. European Commission has proposed a minimum number of recharging points for each Member State based on the number of electric vehicles. At least $10 \%$ of these should be publicly accessible.
TABLE I

TECHNOLOGICAL ALTERNATIVES FOR EV FAST CHARGING STATIONS

\begin{tabular}{ccccc}
\hline & $\begin{array}{c}\text { AC } \\
\text { Charging }\end{array}$ & $\begin{array}{c}\text { Fast DC } \\
\text { Charging }\end{array}$ & $\begin{array}{c}\text { Inductive } \\
\text { Charging }\end{array}$ & $\begin{array}{c}\text { Battery } \\
\text { Swapping }\end{array}$ \\
\hline Power & $44 \mathrm{~kW}$ & $50 \mathrm{~kW}$ & $11 \mathrm{~kW}$ & - \\
\hline Voltage & $400 \mathrm{~V}$ & $400 \mathrm{VDC}$ & $400 \mathrm{~V}$ & - \\
\hline Current & $63 \mathrm{~A}$ & $125 \mathrm{~A}$ & $16 \mathrm{~A}$ & - \\
\hline $\begin{array}{c}\text { Duration } \\
\text { of 20kWh } \\
\text { charge cycle }\end{array}$ & $27 \mathrm{~min}$ & $24 \mathrm{~min}$ & $109 \mathrm{~min}$ & $5 \mathrm{~min}$ \\
\hline
\end{tabular}

Publicly accessible charging facilities should be understood as complementary to private slow charging (typically household or office spots) for promoting EVs for urban and interurban mobility. Publicly available EV fuelling stations should provide short fuelling time, thus fast charging stations seems to be the best option for publicly accessible infrastructure. As it can be seen in Table I there are different technological alternatives for fast charging of electric vehicles including onboard AC fast charging, battery swapping, DC fast charging, and (less fast) inductive charging. Among them, DC fast charging technology is the most widespread, being currently available on several commercial EVs in a separate Mode 4 DC fast charging socket. Connectors allowing for both AC and DC charge at a unique vehicle inlet (termed Combo) are also being studied and proposed for the future.

A network of fast charging stations is needed. However it is still an open question where these charging stations should be placed and how they would be designed and sized [1]. In terms of location it is important to highlight that fast charging stations could have a significant impact on electricity distribution network due to its high power demand ratings. As a consequence building up the grid tie to the distribution network is one of the main costs of the fast charging stations commissioning, together with the equipment (power electronics) and the required space for parking and for equipment location. State of the art fuelling stations location methodologies are mainly dealing with traffic flows and population distribution along the territory (where people live and work). Therefore, classical planning tools applied to fast charging stations could provide sub-optimal solutions in terms of cost 
of installation: location provided by these methodologies could be optimal from the mobility point of view, but very expensive if commissioning costs are not considered. The aim of this paper is to find the optimal location for EV fast charging stations by means of two methodologies: a classical flowcapturing optimization model involving only mobility needs, and a proposed advanced flow-capturing optimization model including fast charging stations costs (distribution network, equipment and location costs). While the first model aims to maximize the public service provided by the fast charging stations, the second also considers their profitability. Results from both models are compared in order to analyse the effect of both planning approaches in terms of total cost. As a case study it has been chosen the city of Barcelona.

\section{STATE OF THE ART OF OPTIMAL LOCATION PROBLEMS}

The literature regarding allocation of fuelling facilities within a net is vast. Two main approaches can be found with different behavioral hypothesis of consumption behind: the so called $p$-median models first developed by Hakimi [2] and Revelle and Swain [3] minimize the distance from the house of the consumers to the facilities; and the fluxes models, that maximize the quantity of fluxes captured by each facility. Taking into account that public facilities should be considered as an alternative for private charging points located at household and office level, fluxes models seem to be best-fitted for publicly available fast charging stations planning.

In the fluxes approach, the Flow-Capturing LocationAllocation Model (FCLM), introduced by Hodgson [4] and developed also by and Berman, Larson and Fouska [5] the objective is to maximize the number of flows captured by each facility and the demand is considered to be satisfied when a facility is located anywhere along the path. Several extensions and applications of the FCLM can be found in Averbakh and Berman [6], Hodgson and Berman [7], [8].

Taking into account limited range of non conventional vehicles such as electric vehicles or hydrogen vehicles, Kuby and Lim [9] formulated the Fuel-Refueling Location- Allocation Model (FRLM). Based on the FCLM, the FRLM counts a flow as refuelled only if a combination of stations exists on a path that can successfully refuel the round trip between the origin and destination, given the assumed driving range of vehicles [10]. The model has been developed and applied in Kuby and Lim [11], Lim and Kuby [12], Kuby, Lines, Schultz, Xie, Kim and Lim [13] and Kuby and Kim [14], among others.

Since EVs have a limited range (aroun 150-200 km) FRLM methodology could be considered for locating publicly available charging stations. However, in the short term it is considered that EVs will be mainly used in urban areas, running less than 50-70 kilometres a day. Therefore classical FCLM methodology can be applied directly, letting FRLM for further stages of electromobility where inter-urban would be widespread.

\section{OPTIMAL LOCATION METHODOLOGY}

As described in the previous section, considering the specific characteristics of electromobility as well as the case study to be analysed, the models to be applied in this project are based on the the Flow-Capturing Location-Allocation Model (FCLM). Thus, based on the original model described above [4], two methodologies are applied and compared:

- Methodology 1: classical Flow-Capturing LocationAllocation optimization model.

- Methodology 2: modified Flow-Capturing LocationAllocation model including fast charging stations installation cost.

On one hand Methodology 1 is focused on locate the facilities so as to capture as many flows as possible. Then, in this model the objective is to maximize the number of flows captured given a fixed number of charging facilities to be locate. In the other hand Methodology 2 aims to locate the facilities so as to capture a fixed amount of flows at the lower cost. Then, in this model the number of charging facilities is optimized instead of introduced as a fixed parameter. In both models the demand is considered to be satisfied when there is a facility anywhere along the path. Both models are in detail described below.

\section{A. Model description: Methodology 1}

Methodology 1 is based on the classical Flow-Capturing Location-Allocation Model (FCLM) [4]. The objective function (1) aims to maximize the amount of flow that can be refuelled using a fixed number $p$ of charging facilities. The binary decision variables of the model are the following:

- $x_{k}$, which is 1 if there is a facility at location $k$, and 0 if not.

- $y_{q}$, binary variable expressing the captured paths; $y_{q}$ is 1 if path $f_{q}$ is captured, 0 if not.

The first set of constrains (2) ensures that flows can only be refuelled if there is at least one open facility in the path to be considered. The constraint (3) requires exactly $p$ fast charging facilities to be installed. The set of constraints defined in (4) ensures that only whole facilities will be located. The last set of constraints (5) prevents the proportion of the flow refuelled from exceeding 1. Both set of constraints (4) and (5) define decision variables as binary.

$$
\begin{array}{lll}
\operatorname{Max} & \sum_{q \in Q} f_{q} y_{q} & \\
\text { s.t } & & \\
& \sum_{k \in N_{q}} x_{k} \geq y_{q} & \forall q \in Q \\
& \sum_{k \in K} x_{k}=p & \\
& x_{k} \in\{0,1\} & \forall k \in K \\
& y_{q} \in\{0,1\} & \forall q \in Q
\end{array}
$$

where: 
$Q \quad$ Set of all $\mathrm{O}-\mathrm{D}$ paths

$K \quad$ Set of all potencial facility locations

$N_{q} \quad$ Set of nodes on path $q$ between $O_{i}$ and $D_{j}$

$f_{q} \quad$ Flow on path $q$ [vehicles/year]

$p \quad$ Number of facilities to be located

\section{B. Model description: Methodology 2}

Methodology 2 is also based on the classical FlowCapturing Location-Allocation Model (FCLM). However, in this case the objective function (6) aims to minimize the total cost of installing the fast charging stations given the minimum amount of flow to be captured $F_{\text {min }}$. As in Methodology 1, the binary decision variables of the model are the following:

- $x_{k}$, which is 1 if there is a facility at location $k$, and 0 if not.

- $y_{q}$, binary variable expressing the captured paths; $y_{q}$ is 1 if path $f_{q}$ is captured, 0 if not.

The first constraint (7) requires exactly a minimum flow to be refuelled. The second set of constrains (8) ensures that flows can only be refuelled if there is at least one open facility in the path to be considered. The third constraint (9) ensures that only whole facilities will be located. The last constraint (10) prevents the proportion of the flow refuelled from exceeding 1. Both set of constraints (9) and (10) define decision variables as binary.

$$
\begin{aligned}
& \text { Min } \quad \sum_{k \in K} c_{k} X_{k} \\
& \text { s.t } \\
& \sum_{q \in Q} f_{q} Y_{q} \geq F_{\min } \\
& \sum_{k \in N_{q}} x_{k} \geq Y_{q} \quad \forall q \in Q \\
& x_{k} \in\{0,1\} \quad \forall k \in K \\
& Y_{q} \in\{0,1\} \quad \forall q \in Q
\end{aligned}
$$

where, beside the notation defined in Methodology 1:

$F_{\text {min }}$ Minimum amount of flow to be captured [vehicles/year] $c_{k} \quad$ Installation cost at location $k$ [€]

\section{CASE STUdy}

As a case study it has been chosen the city of Barcelona. In order to build the commuting paths and the fluxes for the Origin Destination Matrix two sources of information were used. The first one was the Mobility Survey from the Metropolitan Transport Agency for the year 2006, and the second one the Catalonia Roads Graph published by the Government of the Autonomous Community. For estimating the traffic flows through the road network Dijkstra shortest paths algorithm was applied [17].
TABLE II

PERFORMANCE COMPARISON OF METHOD 1 AND METHOD 2

\begin{tabular}{cccc} 
& \multicolumn{3}{c}{ FLOW REFUELLED } \\
\cline { 2 - 4 } & $50 \%$ & $70 \%$ & $92 \%$ \\
\hline Method 1: FCLM & \multicolumn{3}{c}{27} \\
\hline $\begin{array}{c}\text { Number of } \\
\text { fuelling stations }\end{array}$ & 5 & 11 & \\
\hline Total Cost [€] & 517,420 & $1,124,840$ & $2,753,616$ \\
\hline $\begin{array}{c}\text { Method 2: } \\
\text { Cost Minimization }\end{array}$ & & & 28 \\
\hline $\begin{array}{c}\text { Number of } \\
\text { fuelling stations }\end{array}$ & 7 & 12 & $2,589,680$ \\
\hline Total Cost [€] & 481,968 & $1,010,904$ & $\mathbf{6 \%}$ \\
\hline \hline SAVINGS [\%] & $\mathbf{6 . 9 \%}$ & $\mathbf{1 0 . 1 \%}$ & $\mathbf{6}$
\end{tabular}

\section{A. Description of observed area}

This work is mainly based on the mobility survey performed by the Autoritat del Transport Metropolita [15]. Within the survey, the Metropolitan Area of Barcelona is segmented in 305 zones, 63 of which correspond to the city of Barcelona. The simplified version of the roads graph published by the local government Catalonia Roads Graph was the input used to build the shortest paths along the 63 origin destination zones defined in Barcelona. The graph was filtered only with the city of Barcelona information, where there are placed 940 nodes and 2552 edges.

\section{B. Assumptions on mobility}

The data used correspond to the trips made in private vehicle between zones within the city and the commuting trips to Barcelona from the other 242 areas. It has been considered an homogeneous penetration of EVs in each zone of Barcelona. The commuters were assigned to the different zones where the entries to the city are located according to the map of highways of the region.

\section{Installation costs}

The commissioning costs of the fast charging stations of each of the 940 feasible locations was differentiated in four categories according to the following criteria (Figure 1): the existence of a fuel station with car wash (Type 1); fuel station with more than 10 pumps (Type 2); fuel stations with less than 10 pumps and malls and supermarkets (Type 3); and neither of the previous options (Type 4). For that purpose, a map containing all fuel stations, hypermarkets and malls of the city was built and the facilities were assigned to the closest node of the net given its location. The data regarding fuel stations was quoted on-line from the Spanish Ministry of Industry, Tourism and Commerce [16]; the distribution network costs equipment costs were taken from [18], and the costs of localization in Barcelona were assigned according to current average prices of land.

\section{Results}

The models described in the previous sections are integer linear problems that have been formulated using the General Algebraic Modelling System (GAMS) and have been solved using CPLEX with standard options. Both models were used 


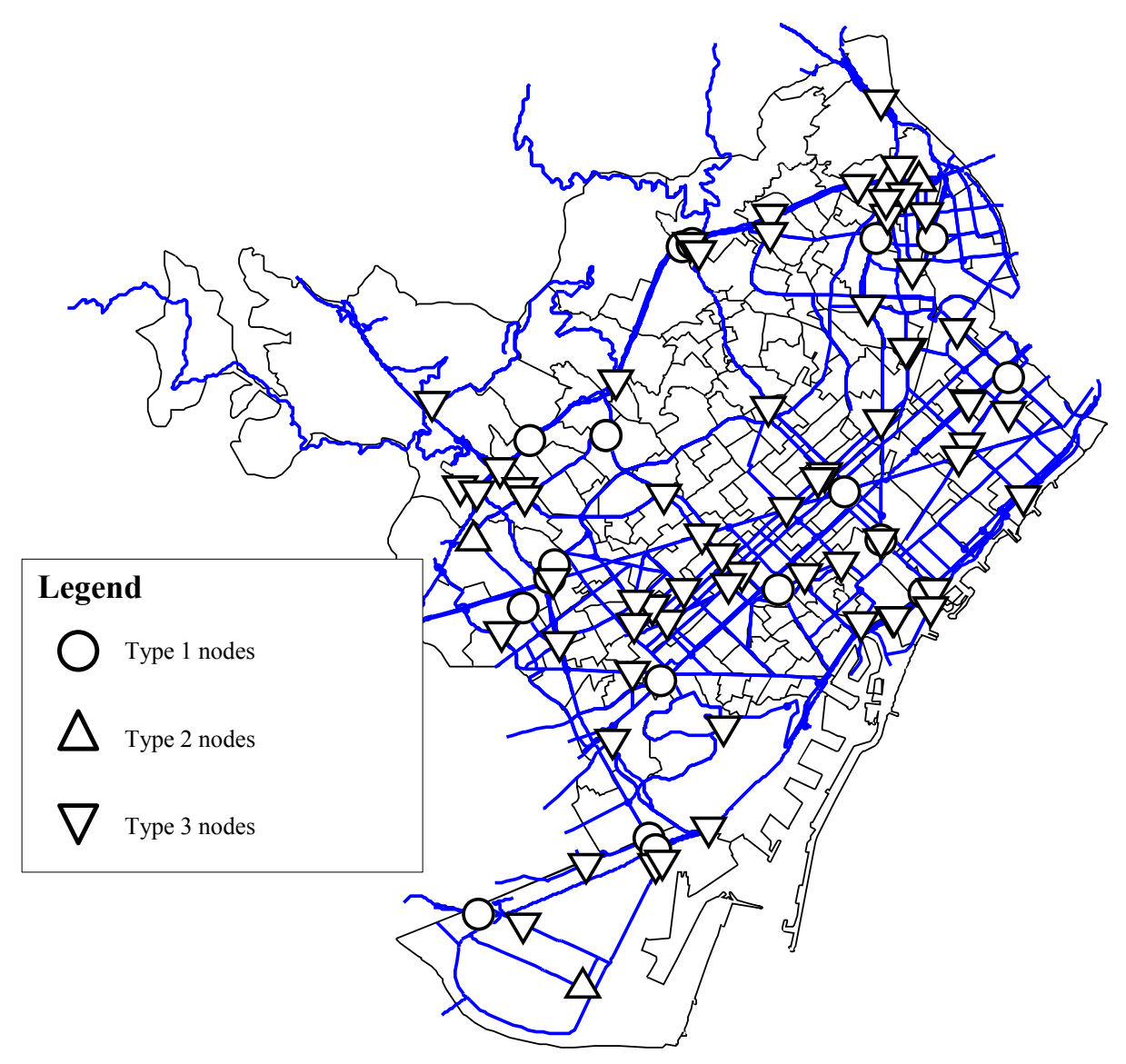

Figure 1. Current location of fuel stations, hypermarkets and malls in Barcelona used for defining fast charging installation costs in the road network nodes

for finding the optimal location of fast charging stations that capture $50 \%, 70 \%$ and $92 \%$ of Barcelona's yearly traffic flows. As a result it was obtained the optimal subset of network nodes for locating the charging facilities and the total cost of installing them. For ensuring that the captured flows with Methodology 1 are $50 \%, 70 \%$ and $92 \%$, the number $p$ of charging stations is increased until the captured flow exceeds these values.

In Figure 2 results from both methodologies for capturing $50 \%$ of Barcelona's yearly traffic flows are represented. As it can be seen in the figure, two fast charging stations were located in the same place for both Methodology 1 and Methodology 2. Three out of the five fast charging stations initially located in the southern part of Barcelona by Methodology 1, were reallocated by Methodology 2 in the northern part, were two of the main access highways enter to Barcelona. Furthermore two fast charging stations were located by Methodology 2 additionally to the ones located by Methodology 1. All new fast charging stations were located in network nodes currently housing a fuel station. Three of them contained car wash services, reducing considerably electricity distribution grid reinforcements.
It can be seen how the total number of required charging stations for capturing $50 \%$ of Barcelona's yearly traffic is higher for Methodology 2, increasing from 5 to 7 . However, as it can be appreciated in Table II the total cost for locating them is reduced a $7 \%$. The cost reduction from Methodology 2 has been obtained as a consequence of using those locations with lower installation costs, i.e. those locations were currently there is a fuel station, an hypermarket or a mall, despite the total number of facilities is greater.

In Table II the total number of required charging stations as well as the total cost of installing them is compared for both methodologies and different traffic flow refuelling targets. For all cases it can be seen how the total number of charging stations needed for supplying a certain level of demand is lower for Methodology 1. However, in terms of cost, it can be seen how savings in the range from $5 \%$ to $10 \%$ are achieved by applying Methodology 2, where cost minimization is pursued. Savings obtained by the application of Methodology 2 in other cities will depend on the mobility matrix and the current location of fuelling stations and publicly available parking lots. 


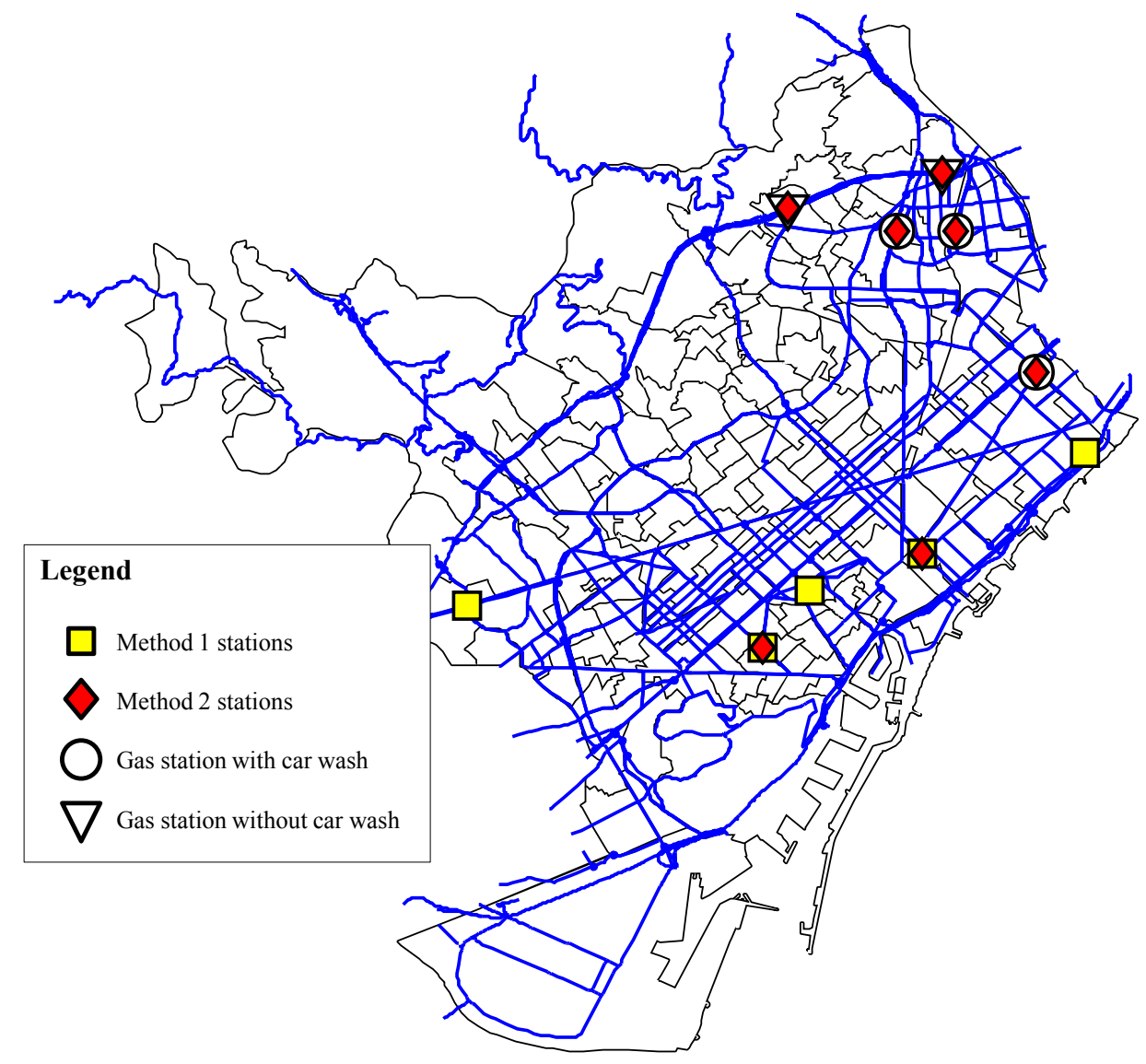

Figure 2. Map of Barcelona fast charging stations network provided by Method 1 (yellow) and Method 2 (red) with $50 \%$ of the traffic flows refuelled

\section{CONCLUSIONS}

Availability of recharging stations is not only a technical prerequisite for the functioning of electric vehicles, but also one of the most critical components of consumer acceptance. Fast charging stations seem to be the best option for publicly accessible infrastructure, taking into account length of the charging process, upfront costs and market availability. However, space limitations and distribution grid congestion within urban areas increases installation costs of that kind of facilities. Fast charging location planning according to classical Flow-Capturing Location-Allocation Models render a set of locations that does not take into account variance in set-up costs, being only focused on maximizing the refuelled traffic flows for a fixed number of charging facilities. With the methodology proposed in this work the set-up costs of the fast charging stations in Barcelona's road network were also considered, using those locations currently housing a fuel station, an hypermarket or a mall, and therefore reducing location costs and distribution network reinforcing costs. Tension between planning objectives regarding charging stations accessibility and sound project finance are solved with the proposed methodology, reaching a cost reduction in the range of $5 \%$ to $10 \%$. The proposed methodology is easily replicable to other cities, were savings obtained will depend on the mobility matrix and the current location of fuelling stations and publicly available parking lots.

\section{REFERENCES}

[1] Corchero, C.; Cruz-Zambrano, M.; Heredia, F.-J.; Cairo, J.-I.; IgualadaGonzalez, L.; Romero-Ortega, A.; , "Optimal sizing of microgrids: A fast charging station case," European Energy Market (EEM), 2012 9th International Conference on the , vol., no., pp.1-6, 10-12 May 2012 doi: 10.1109/EEM.2012.6254677

[2] Hakimi, S.L. (1964) Optimum locations of switching centres and the absolute centres and medians of a grap., Operations Research 12, 450459.

[3] Revelle, C.S. and R. Swain (1970) Central facilities location, Geographical Analysis 2, 3042.

[4] Hodgson, M.J. (1990) A flow capturing location allocation model, Geographical Analysis 22, 270279.

[5] Berman, O., Larson, R.C., N. Fouska (1992) Optimal location of discretionary service facilities, Transportation Science 26, 201211.

[6] Averbakh, I., and O. Berman (1996) Locating ow-capturing units on a network with multi-counting and diminishing returns to scale, European Journal of Operational Research 91, 495506.

[7] Hodgson, M.J., and O. Berman (1997) A billboard location model, Geographical and Environment Modeling 1, 2545.

[8] Hodgson, M.J., K.E. Rosing, and J. Zhang (1996) Locating vehicle inspection station to protect a transportation network, Geographical Analysis 28, 299314. 
[9] Kuby, M.J. and S. Lim (2005) The flow-refueling location problem for alternative-fuel vehicles, Socio-Economic Planning Sciences 39, 125145.

[10] Upchurch, C. Kuby, M. Comparing the p-median and flow-refueling models for locating alternative-fuel stations, Journal of Transport Geography, Volume 18, Issue 6, November 2010, Pages 750-758, ISSN 09666923, 10.1016/j.jtrangeo.2010.06.015.

[11] Kuby, M. and S. Lim (2007) Location of alternative-fuel stations using the flow-refueling location model and dispersion of candidate sites on arcs, Networks and Spatial Economics 7 (2), 129152

[12] Lim, S., Kuby, M. (2010) Heuristic algorithms for siting alternativefuel stations using the flow-refueling location model, European Journal of Operational Research 204 (1), 5161.

[13] Kuby, M., Lines, L., Schultz, R., Xie, Z., Kim, J. and S. Lim (2009) Optimal location strategy for hydrogen refueling stations in Florida, International Journal of Hydrogen Energy 34, 60456064.

[14] Kuby and Kim (2012) The deviation-flow refueling location model for optimizing a network of refueling stations, International Journal of Hydrogen Energy vo. 3 7, pages $5406-5420$

[15] Enquesta Mobilitat Quotidiana de Catalunya (EMQ 2006). Autoritat del Transport Metropolit i el Departament de Poltica Territorial i Obres Pbliques de la Generalitat de Catalunya.

[16] Spanish Ministry of Industry, Tourism and Commerce, http://www.mityc.es/, accessed on 2012-09-01.

[17] Dijkstra, E. W. A note on two problems in connexion with graphs, Numerische Mathematik, 1959, Volume 1, Issue 1, pp 269-271

[18] Andreas Schroeder, Thure Traber, The economics of fast charging infrastructure for electric vehicles, Energy Policy, Volume 43, April 2012, Pages 136-144, ISSN 0301-4215, 10.1016/j.enpol.2011.12.041. 